\title{
Vol. 66, No. 18
}

In the report "State HCV Incidence and Policies Related to HCV Preventive and Treatment Services for Persons Who Inject Drugs - United States, 2015-2016," on page 466, the second sentence of the second paragraph should have read "HCV incidence rates increased by $167 \%$ nationally from 0.3 cases per $\mathbf{1 0 0 , 0 0 0}$ U.S. population in 2010 to 0.8 in 2015 (4)."

In the cover box "Hepatitis Awareness Month and Testing Day - May 2017," on page 465, the last sentence of the second paragraph should have read "During 2010-2015, HCV incidence rates increased by $167 \%$ with the highest rates among young persons who inject drugs (PWID). †” 